\title{
The Alternating Direction Implicit Body of Revolution Multiresolution Time Domain Method with Convolution Perfect Matched Layer
}

\author{
Chen Yiwang, ${ }^{1}$ Ni Jiazheng, ${ }^{1}$ Liu Yawen, ${ }^{2}$ Dong Xiaohua, ${ }^{1}$ and Zhang Pin ${ }^{1}$ \\ ${ }^{1}$ National Key Laboratory on Electromagnetic Environment and Electro-Optical Engineering, \\ PLA University of Science and Technology, Nanjing, Jiangsu 210007, China \\ ${ }^{2}$ Department of Transportation Service, Bengbu Automobile N.C.O. Academy of PLA, Bengbu, Anhui 233010, China
}

Correspondence should be addressed to Ni Jiazheng; nijiazheng501@163.com

Received 27 September 2016; Revised 8 December 2016; Accepted 4 January 2017; Published 13 February 2017

Academic Editor: Nazrul Islam

Copyright (C) 2017 Chen Yiwang et al. This is an open access article distributed under the Creative Commons Attribution License, which permits unrestricted use, distribution, and reproduction in any medium, provided the original work is properly cited.

\begin{abstract}
Overmuch memory and time of CPU have been taken by multiresolution time domain (MRTD) method in three-dimension issues. In order to solve this problem, the alternating direction implicit body of revolution multiresolution time domain (ADI-BORMRTD) scheme is presented. Firstly, based on body of revolution finite difference time domain (BOR-FDTD) method, equations of body of revolution multiresolution time domain (BOR-MRTD) method are implemented. Then alternating direction implicit (ADI) is introduced into BOR-MRTD method. Lastly, convolution perfect matched layer (CPML) is applied for ADI-BOR-MRTD method. Numerical results demonstrate that ADI-BOR-MRTD method saves more memory and time of CPU than FDTD and MRTD methods.
\end{abstract}

\section{Introduction}

As an efficient numerical algorithm, the multiresolution time domain (MRTD) method was applied in electromagnetic field computation in 1996 by Krumpholz and Katehi [1] firstly. Compared with the finite difference time domain (FDTD) method, the MRTD method has lower numerical dispersion and saves more memory and time of $\mathrm{CPU}$ $[1,2]$.

The time index and the calculating efficiency of the MRTD method are generally limited by the CourantFriedrich-Levy (CFL) stability condition. However, the alternating direction implicit (ADI) technique can overcome the CFL limitation [3]. Chen and Zhang had published the ADI-MRTD scheme in 2001 [4]. The time step size for the ADI-MRTD is only limited by modeling accuracy of the calculation. Then, the study on the numerical dispersion, absorbing boundary conditions, and the application in the one-dimension photoelectronic band-gap of the ADI-MRTD scheme are developed gradually [5-7].
The body of revolution is an important target in electromagnetic field computation. In order to calculate the body of revolution with less memory and time of CPU, the ADIBOR-MRTD scheme is presented. At the end of the work, the convolution perfect matched layer (CPML) formulations are derived for the ADI-BOR-MRTD scheme.

\section{Equations of BOR-MRTD}

In cylindrical coordinates, Maxwell's equations should be written as

$$
\begin{aligned}
& \varepsilon \frac{\partial E_{\rho}}{\partial t}+\sigma E_{\rho}=\frac{1}{\rho} \frac{\partial H_{z}}{\partial \varphi}-\frac{\partial H_{\varphi}}{\partial z} \\
& \varepsilon \frac{\partial E_{\varphi}}{\partial t}+\sigma E_{\varphi}=\frac{\partial H_{\rho}}{\partial z}-\frac{\partial H_{z}}{\partial \rho} \\
& \varepsilon \frac{\partial E_{z}}{\partial t}+\sigma E_{z}=\frac{1}{\rho} \frac{\partial\left(\rho H_{\varphi}\right)}{\partial \rho}-\frac{1}{\rho} \frac{\partial H_{\rho}}{\partial \varphi}
\end{aligned}
$$




$$
\begin{aligned}
-\mu \frac{\partial H_{\rho}}{\partial t}-\sigma_{m} H_{\rho} & =\frac{1}{\rho} \frac{\partial E_{z}}{\partial \varphi}-\frac{\partial E_{\varphi}}{\partial z} \\
-\mu \frac{\partial H_{\varphi}}{\partial t}-\sigma_{m} H_{\varphi} & =\frac{\partial E_{\rho}}{\partial z}-\frac{\partial E_{z}}{\partial \rho} \\
-\mu \frac{\partial H_{z}}{\partial t}-\sigma_{m} H_{z} & =\frac{1}{\rho} \frac{\partial\left(\rho E_{\varphi}\right)}{\partial \rho}-\frac{1}{\rho} \frac{\partial E_{\rho}}{\partial \varphi} .
\end{aligned}
$$

The electric and magnetic fields are expanded by Fourier series as

$$
\begin{aligned}
& \mathbf{E}=\sum_{m=0}^{\infty}\left(\mathbf{e}_{u} \cos m \phi+\mathbf{e}_{v} \sin m \phi\right) \\
& \mathbf{H}=\sum_{m=0}^{\infty}\left(\mathbf{h}_{u} \cos m \phi+\mathbf{h}_{v} \sin m \phi\right),
\end{aligned}
$$

where $\mathbf{e}_{u}, \mathbf{e}_{v}, \mathbf{h}_{u}, \mathbf{h}_{v}$ are Fourier coefficients and $\mathbf{e}=\mathbf{E}_{m}, \mathbf{h}=$ $\mathbf{H}_{m} . \phi$ is azimuth angle; $m$ is modulus. $u$ is related to $\cos m \phi$; $v$ is related to $\sin m \phi$.

Substituting (2a) and (2b) to (1a)-(1f), (1a)-(1f) are rewritten as

$$
\begin{aligned}
\varepsilon \frac{\partial e_{\rho}}{\partial t} & = \pm \frac{m}{\rho} h_{z}-\frac{\partial h_{\varphi}}{\partial z} \\
\varepsilon \frac{\partial e_{\varphi}}{\partial t} & =\frac{\partial h_{\rho}}{\partial z}-\frac{\partial h_{z}}{\partial \rho} \\
\varepsilon \frac{\partial e_{z}}{\partial t} & =\frac{1}{\rho} \frac{\partial\left(\rho h_{\varphi}\right)}{\partial \rho} \mp \frac{m}{\rho} h_{\rho} \\
\mu \frac{\partial h_{\rho}}{\partial t} & = \pm \frac{m}{\rho} e_{z}+\frac{\partial e_{\varphi}}{\partial z} \\
\mu \frac{\partial h_{\varphi}}{\partial t} & =-\frac{\partial e_{\rho}}{\partial z}+\frac{\partial e_{z}}{\partial \rho} \\
\mu \frac{\partial h_{z}}{\partial t} & =-\frac{1}{\rho} \frac{\partial\left(\rho e_{\varphi}\right)}{\partial \rho} \mp \frac{m}{\rho} e_{\rho} .
\end{aligned}
$$

The electric and magnetic fields are expanded by Daubechies' scaling function in space domain and by Haar's scaling function in time domain.

$$
\begin{gathered}
e_{\rho}(\vec{r}, t)=\sum_{i, j, k, n=-\infty}^{+\infty} e_{i+1 / 2, j, k}^{\alpha \rho, n} \Phi_{n}(t) \alpha_{i+1 / 2}(\rho) \alpha_{j}(\varphi) \\
\alpha_{k}(z) \\
e_{\varphi}(\vec{r}, t)=\sum_{i, j, k, n=-\infty}^{+\infty} e_{i, j+1 / 2, k}^{\alpha \varphi, n} \Phi_{n}(t) \alpha_{i}(\rho) \alpha_{j+1 / 2}(\varphi) \\
\cdot \alpha_{k}(z) e_{z}(\vec{r}, t)=\sum_{i, j, k, n=-\infty}^{+\infty} e_{i, j, k+1 / 2}^{\alpha z, n} \Phi_{n}(t) \alpha_{i}(\rho) \alpha_{j}(\varphi) \\
\cdot \alpha_{k+1 / 2}(z)
\end{gathered}
$$

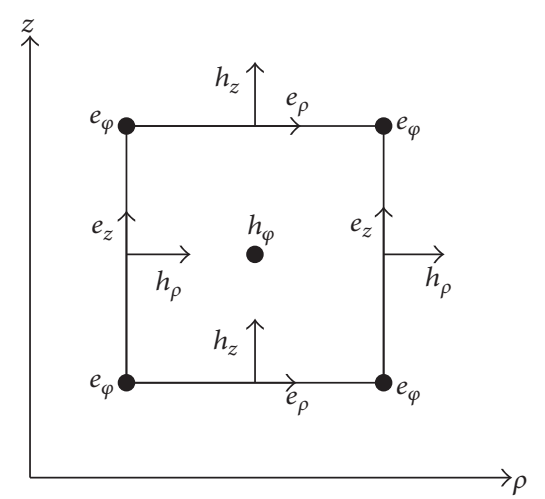

FIGURE 1: Distribution of field components for BOR-MRTD.

$$
\begin{aligned}
& h_{\rho}(\vec{r}, t)=\sum_{i, j, k, n=-\infty}^{+\infty} h_{i, j+1 / 2, k+1 / 2}^{\alpha \rho, n+1 / 2} \Phi_{n+1 / 2}(t) \alpha_{i}(\rho) \\
& \cdot \alpha_{j+1 / 2}(\varphi) \alpha_{k+1 / 2}(z) \\
& h_{\varphi}(\vec{r}, t)=\sum_{i, j, k, n=-\infty}^{+\infty} h_{i+1 / 2, j, k+1 / 2}^{\alpha \varphi, n+1 / 2} \Phi_{n+1 / 2}(t) \alpha_{i+1 / 2}(\rho) \\
& \cdot \alpha_{j}(\varphi) \alpha_{k+1 / 2}(z) \\
& h_{z}(\vec{r}, t)=\sum_{i, j, k, n=-\infty}^{+\infty} h_{i+1 / 2, j+1 / 2, k}^{\alpha z, n+1 / 2} \Phi_{n+1 / 2}(t) \alpha_{i+1 / 2}(\rho) \\
& \cdot \alpha_{j+1 / 2}(\varphi) \alpha_{k}(z) .
\end{aligned}
$$

$e_{i, j, k}^{\alpha \zeta, n}$ and $h_{i, j, k}^{\alpha \zeta, n}$ are the field coefficients, with $\zeta=\rho, \varphi, z$. The indexes $i, j, k$, and $n$ are the space indices and time indices as $\rho=i \Delta \rho, \varphi=j \Delta \varphi, z=k \Delta z$, and $t=n \Delta t$, where $\Delta \rho, \Delta \varphi, \Delta z$, and $\Delta t$ represent the space and time discretization intervals in $\rho^{-}, \varphi^{-}, z^{-}$, and $t$-direction. The function $\Phi(t)$ is Haar's scaling function [8], and $\alpha$ is Daubechies' scaling function [9].

The distribution of field components is shown in Figure 1.

The equations of BOR-MRTD method are presented as follows:

$$
\begin{gathered}
e_{\rho}^{n+1}\left(i+\frac{1}{2}, k\right)=e_{\rho}^{n}\left(i+\frac{1}{2}, k\right) \pm \frac{m \Delta t}{(i+1 / 2) \varepsilon \Delta \rho} \\
\cdot h_{z}^{n+1 / 2}\left(i+\frac{1}{2}, k\right)-\frac{\Delta t}{\varepsilon \Delta z} \\
\cdot \sum_{l} a(l) h_{\varphi}^{n+1 / 2}\left(i+\frac{1}{2}, k+l+\frac{1}{2}\right) \\
e_{\varphi}^{n+1}(i, k)=e_{\varphi}^{n}(i, k)+\frac{\Delta t}{\varepsilon \Delta z} \\
\cdot \sum_{l} a(l) h_{\rho}^{n+1 / 2}\left(i, k+l+\frac{1}{2}\right)-\frac{\Delta t}{\varepsilon \Delta \rho} \\
\cdot \sum_{l} a(l) h_{z}^{n+1 / 2}\left(i+l+\frac{1}{2}, k\right) .
\end{gathered}
$$


TABLE 1: The coefficients $a(l)$ of $D_{2}$.

\begin{tabular}{lc}
\hline$l$ & $a(l)$ \\
\hline 0 & 1.22916661202745 \\
1 & -0.09374997764746 \\
2 & 0.01041666418309 \\
\hline
\end{tabular}

$$
\begin{aligned}
& e_{z}^{n+1}\left(i, k+\frac{1}{2}\right)=e_{z}^{n}\left(i, k+\frac{1}{2}\right) \mp \frac{m \Delta t}{\varepsilon i \Delta \rho} h_{\rho}^{n+1 / 2}(i, k \\
& \left.+\frac{1}{2}\right)+\frac{\Delta t}{i \varepsilon \Delta \rho} \sum_{l} a(l)\left(i+l+\frac{1}{2}\right) h_{\varphi}^{n+1 / 2}\left(i+l+\frac{1}{2}, k\right. \\
& \left.+\frac{1}{2}\right) \\
& h_{\rho}^{n+1 / 2}\left(i, k+\frac{1}{2}\right)=h_{\rho}^{n-1 / 2}\left(i, k+\frac{1}{2}\right) \\
& +\frac{\Delta t}{\mu}\left(\frac{1}{\Delta z} \sum_{l} a(l) e_{\varphi}^{n}(i, k+l+1)\right. \\
& \left. \pm \frac{m}{i \Delta \rho} e_{z}^{n}\left(i, k+\frac{1}{2}\right)\right) \\
& h_{\varphi}^{n+1 / 2}\left(i+\frac{1}{2}, k+\frac{1}{2}\right)=h_{\varphi}^{n-1 / 2}\left(i+\frac{1}{2}, k+\frac{1}{2}\right)+\frac{\Delta t}{\mu \Delta \rho} \\
& \cdot \sum_{l} a(l) e_{z}^{n}\left(i, k+l+\frac{1}{2}\right)-\frac{\Delta t}{\mu \Delta z} \\
& \cdot \sum_{l} a(l) e_{\rho}^{n}\left(i+\frac{1}{2}, k+l+1\right) \\
& h_{z}^{n+1 / 2}\left(i+\frac{1}{2}, k\right)=h_{z}^{n-1 / 2}\left(i+\frac{1}{2}, k\right) \\
& +\frac{\Delta t}{\mu(i+1 / 2) \Delta \rho}\left(\mp m e_{\rho}^{n}\left(i+\frac{1}{2}, k\right)\right. \\
& \left.-\sum_{l} a(l)(i+l+1) e_{\varphi}^{n}(i+l+1, k)\right) .
\end{aligned}
$$

The coefficient $a(l)$ is equal to

$$
\begin{aligned}
a(l) & \equiv\left\langle\frac{\partial \alpha_{m^{\prime}+1 / 2}(x)}{\partial x}, \alpha_{m-1}(x)\right\rangle \\
& =\frac{1}{\pi} \int_{0}^{\infty}|\widehat{\alpha}(\omega)|^{2} \sin [\omega(l+0.5)] d \omega .
\end{aligned}
$$

For Daubechies' scaling function with two vanishing moments $\left(D_{2}\right)$, the coefficients are shown in Table 1; for $l>2, a(l)$ are zeros due to the compact support of Daubechies' scaling function; for $l<0, a(l)$ are given by the symmetry relation $a(-1-l)=-a(l)$.
When $\rho=0$, namely, $i=0$, the values of $e_{\phi}, e_{z}, h_{\rho}$ are singular. It can be solved via Ampere's law:

$$
\oint_{\mathcal{c}} \mathbf{H} \cdot d \mathbf{l}=\varepsilon \frac{\partial}{\partial t} \iint_{s} \mathbf{E} \cdot d \mathbf{s}+\iint_{s} \sigma \mathbf{E} \cdot d \mathbf{s}
$$

Taking the integral of (7),

$$
\begin{aligned}
e_{z}^{n+1}\left(0, k+\frac{1}{2}\right)= & e_{z}^{n}\left(0, k+\frac{1}{2}\right) \\
& +\frac{4 \Delta t}{\varepsilon \Delta \rho} h_{\varphi}^{n+1 / 2}\left(\frac{1}{2}, k+\frac{1}{2}\right), \quad m=0 .
\end{aligned}
$$

According to distribution of $e_{\varphi}, e_{z}, h_{\rho}$, when $m \neq 1$, $h_{\rho}(0, k+1 / 2)=e_{\varphi}(0, k)=0$, and when $m>0, e_{z}(0, k+1 / 2)=$ 0 . When $\rho=0$, it is not necessary to calculate $e_{\varphi}$, because the coefficient of $e_{\varphi}(0, k)$ is 0 in (5f). $h_{\rho}(0, k+1 / 2)$ is just useful to calculate $e_{\varphi}(0, k)$, so it is needless too. The conclusion is that $e_{z}$ with $m=0$ is the only field component to be calculated when $\rho=0$.

\section{Equations of ADI-BOR-MRTD}

In alternating direction implicit method, field components have been calculated at $t=n \Delta t$ and $t=(n+1 / 2) \Delta t$. The time has been discretized in two steps, namely, $n \rightarrow n+1 / 2$ and $n+1 / 2 \rightarrow n+1$ [10]. The ADI-BOR-MRTD equations are presented as follows.

First Step $(n \rightarrow n+1 / 2)$

$$
\begin{aligned}
& e_{\rho}^{n+1 / 2}\left(i+\frac{1}{2}, k\right)=e_{\rho}^{n}\left(i+\frac{1}{2}, k\right) \\
& +\frac{\Delta t}{2 \varepsilon}\left( \pm \frac{m}{(i+1 / 2) \Delta \rho} h_{z}^{n+1 / 2}\left(i+\frac{1}{2}, k\right)\right. \\
& \left.\quad-\frac{1}{\Delta z} \sum_{l} a(l) h_{\varphi}^{n}\left(i+\frac{1}{2}, k+l+\frac{1}{2}\right)\right) \\
& e_{\varphi}^{n+1 / 2}(i, k)=e_{\varphi}^{n}(i, k) \\
& +\frac{\Delta t}{2 \varepsilon}\left(\frac{1}{\Delta z} \sum_{l} a(l) h_{\rho}^{n+1 / 2}\left(i, k+l+\frac{1}{2}\right)\right. \\
& \left.\quad-\frac{1}{\Delta \rho} \sum_{l} a(l) h_{z}^{n}\left(i+l+\frac{1}{2}, k\right)\right) \\
& \left.+\frac{m}{i \Delta \rho} h_{\rho}^{n}\left(i, k+\frac{1}{2}\right)\right) \\
& e_{z}^{n+1 / 2}\left(i, k+\frac{1}{2}\right)=e_{z}^{n}\left(i, k+\frac{1}{2}\right) \\
& +\frac{\sum_{l} a(l)(i+l+1 / 2) h_{\varphi}^{n+1 / 2}(i+l+1 / 2, k+1 / 2)}{i \Delta \rho}
\end{aligned}
$$




$$
\begin{aligned}
& h_{\rho}^{n+1 / 2}\left(i, k+\frac{1}{2}\right)=h_{\rho}^{n}\left(i, k+\frac{1}{2}\right) \\
& +\frac{\Delta t}{2 \mu}\left(\frac{\sum_{l} a(l) e_{\varphi}^{n+1 / 2}(i, k+l+1)}{\Delta z}\right. \\
& \left. \pm \frac{m}{i \Delta \rho} e_{z}^{n}\left(i, k+\frac{1}{2}\right)\right) \\
& h_{\varphi}^{n+1 / 2}\left(i+\frac{1}{2}, k+\frac{1}{2}\right)=h_{\varphi}^{n}\left(i+\frac{1}{2}, k+\frac{1}{2}\right) \\
& +\frac{\Delta t}{2 \mu}\left(\frac{\sum_{l} a(l) e_{z}^{n+1 / 2}(i+l+1, k+1 / 2)}{\Delta \rho}\right. \\
& \left.\quad-\frac{\sum_{l} a(l) e_{\rho}^{n}(i+1 / 2, k+l+1)}{\Delta z}\right) \\
& h_{z}^{n+1 / 2}\left(i+\frac{1}{2}, k\right)=h_{z}^{n}\left(i+\frac{1}{2}, k\right) \\
& +\frac{\Delta t}{2(i+1 / 2) \mu \Delta \rho}\left(\mp m e_{\rho}^{n+1 / 2}\left(i+\frac{1}{2}, k\right)\right. \\
& \left.-\sum_{l} a(l)(i+l+1) e_{\varphi}^{n}(i+l+1, k)\right) .
\end{aligned}
$$

$$
\text { Second Step }(n+1 / 2 \rightarrow n+1)
$$

$$
\begin{aligned}
& e_{\rho}^{n+1}\left(i+\frac{1}{2}, k\right)=e_{\rho}^{n+1 / 2}\left(i+\frac{1}{2}, k\right) \\
& +\frac{\Delta t}{2 \varepsilon}\left( \pm \frac{m}{(i+1 / 2) \Delta \rho} h_{z}^{n+1 / 2}\left(i+\frac{1}{2}, k\right)\right. \\
& \left.\quad-\frac{1}{\Delta z} \sum_{l} a(l) h_{\varphi}^{n+1}\left(i+\frac{1}{2}, k+l+\frac{1}{2}\right)\right) \\
& e_{\varphi}^{n+1}(i, k)=e_{\varphi}^{n+1 / 2}(i, k) \\
& +\frac{\Delta t}{2 \varepsilon}\left(\frac{1}{\Delta z} \sum_{l} a(l) h_{\rho}^{n+1 / 2}\left(i, k+l+\frac{1}{2}\right)\right. \\
& \left.\quad-\frac{1}{\Delta \rho} \sum_{l} a(l) h_{z}^{n+1}\left(i+l+\frac{1}{2}, k\right)\right) \\
& \left.\quad+\frac{m}{i \Delta \rho} e_{z}^{n+1}\left(i, k+\frac{1}{2}\right)\right) \\
& \quad+\frac{\Delta t}{2 \mu}\left(\frac{\sum_{l} a(l) e_{\varphi}^{n+1 / 2}(i, k+l+1)}{\Delta+k}\left(\frac{\left.\frac{1}{2}\right)=e_{z}^{n+1 / 2}\left(i, k+\frac{1}{2}\right)}{i \Delta \rho} h_{\rho}^{n+1}\left(i, k+\frac{1}{2}\right)\right)\right. \\
& h_{\rho}^{n+1}\left(i, k+\frac{1}{2}\right)=h_{\rho}^{n+1 / 2}\left(i, k+\frac{1}{2}\right) \\
& \quad(i+l+1 / 2) h_{\varphi}^{n+1 / 2}(i+l+1 / 2, k+1 / 2) \\
& i \Delta \rho
\end{aligned}
$$

$$
\begin{array}{r}
h_{\varphi}^{n+1}\left(i+\frac{1}{2}, k+\frac{1}{2}\right)=h_{\varphi}^{n+1 / 2}\left(i+\frac{1}{2}, k+\frac{1}{2}\right) \\
+\frac{\Delta t}{2 \mu}\left(\frac{\sum_{l} a(l) e_{z}^{n+1 / 2}(i+l+1, k+1 / 2)}{\Delta \rho}\right. \\
\left.\quad-\frac{\sum_{l} a(l) e_{\rho}^{n+1}(i+1 / 2, k+l+1)}{\Delta z}\right) \\
h_{z}^{n+1}\left(i+\frac{1}{2}, k\right)=h_{z}^{n+1 / 2}\left(i+\frac{1}{2}, k\right) \\
+\frac{\Delta t}{2(i+1 / 2) \mu \Delta \rho}\left(\mp m e_{\rho}^{n+1 / 2}\left(i+\frac{1}{2}, k\right)\right. \\
\left.\quad-\sum_{l} a(l)(i+l+1) e_{\varphi}^{n+1}(i+l+1, k)\right) .
\end{array}
$$

Equations (9a)-(9f) and (10a)-(10f) can be solved by the generalized Thomas method [5].

When $\rho=0$, namely, $i=0$, the values of $e_{z}$ are singular as BOR-MRTD scheme. So $e_{z}$ is calculated as follows:

$$
\begin{gathered}
(n \rightarrow n+1 / 2) \\
e_{z}^{n+1 / 2}\left(0, k+\frac{1}{2}\right)-\frac{\Delta t^{2}}{\mu \varepsilon \Delta \rho^{2}} \sum_{l} a(l) e_{z}^{n+1 / 2}\left(l+1, k+\frac{1}{2}\right) \\
=e_{z}^{n}\left(0, k+\frac{1}{2}\right)+\frac{2 \Delta t}{\varepsilon \Delta \rho} h_{\varphi}^{n}\left(\frac{1}{2}, k+\frac{1}{2}\right) \\
\quad-\frac{\Delta t^{2}}{\mu \varepsilon \Delta \rho \Delta z} \sum_{l} a(l) e_{\rho}^{n}\left(\frac{1}{2}, k+l+1\right) . \\
(n+1 / 2 \rightarrow n+1) \quad+\frac{2 \Delta t}{\varepsilon \Delta \rho} h_{\varphi}^{n+1 / 2}\left(\frac{1}{2}, k+\frac{1}{2}\right) .
\end{gathered}
$$

\section{Convolution Perfect Matched Layer}

Based on equations of ADI-BOR-MRTD scheme, we can present equations of CPML with consulting paper [11].

$$
\begin{aligned}
& (n \rightarrow n+1 / 2) \\
& e_{\rho}^{n+1 / 2}\left(i+\frac{1}{2}, k\right)=C A_{\rho}\left(i+\frac{1}{2}, k\right) e_{\rho}^{n}\left(i+\frac{1}{2}, k\right) \\
& \quad+C B_{\rho}\left(i+\frac{1}{2}, k\right)\left( \pm \frac{m}{(i+1 / 2) \Delta \rho} h_{z}^{n+1 / 2}\left(i+\frac{1}{2}, k\right)\right. \\
& \quad-\frac{1}{\kappa_{k} \Delta z} \sum_{l} a(l) h_{\varphi}^{n}\left(i+\frac{1}{2}, k+l+\frac{1}{2}\right) \\
& \left.\quad-\psi_{e_{\rho z}^{n}}^{n}\left(i+\frac{1}{2}, k\right)\right)
\end{aligned}
$$




$$
\begin{aligned}
& \psi_{e_{\rho z}}^{n+1 / 2}\left(i+\frac{1}{2}, k\right)=Q_{k} \psi_{e_{\rho z}}^{n}\left(i+\frac{1}{2}, k\right)+\frac{P_{k}}{\Delta z} \\
& \cdot \sum_{l} a(l) h_{\varphi}^{n}\left(i+\frac{1}{2}, k+l+\frac{1}{2}\right) \\
& e_{\phi}^{n+1 / 2}(i, k)=C A_{\varphi}(i, k) e_{\varphi}^{n}(i, k)+C B_{\varphi}(i, k) \\
& \cdot\left(\frac{1}{\kappa_{k} \Delta z} \sum_{l} a(l) h_{\rho}^{n+1 / 2}\left(i, k+l+\frac{1}{2}\right)\right. \\
& -\frac{1}{\kappa_{i} \Delta \rho} \sum_{l} a(l) h_{z}^{n}\left(i+l+\frac{1}{2}, k\right)+\psi_{e_{\varphi z}}^{n+1 / 2}(i, k) \\
& \left.-\psi_{e_{\varphi \rho}}^{n}(i, k)\right) \\
& \psi_{e_{\varphi z}}^{n+1 / 2}(i, k)=Q_{k} \psi_{e_{\varphi z}}^{n}(i, k)+\frac{P_{k}}{\Delta z} \\
& \cdot \sum_{l} a(l) h_{\rho}^{n+1 / 2}\left(i, k+l+\frac{1}{2}\right) \\
& \psi_{e_{\varphi \rho}}^{n+1 / 2}(i, k)=Q_{i} \psi_{e_{\varphi \rho}}^{n}(i, k)+\frac{P_{i}}{\Delta \rho} \\
& \cdot \sum_{l} a(l) h_{z}^{n}\left(i+l+\frac{1}{2}, k\right) \\
& e_{z}^{n+1 / 2}\left(i, k+\frac{1}{2}\right)=C A_{z}\left(i, k+\frac{1}{2}\right) e_{z}^{n}\left(i, k+\frac{1}{2}\right) \\
& +C B_{z}\left(i, k+\frac{1}{2}\right) \\
& \cdot\left(\frac{\sum_{l} a(l)(i+l+1 / 2) h_{\varphi}^{n+1 / 2}(i+l+1 / 2, k+1 / 2)}{i \kappa_{i} \Delta \rho}\right. \\
& \left.\mp \frac{m}{i \Delta \rho} h_{\rho}^{n}\left(i, k+\frac{1}{2}\right)+\psi_{e_{z \rho}}^{n+1 / 2}\left(i, k+\frac{1}{2}\right)\right) \\
& \psi_{e_{z \rho}}^{n+1 / 2}\left(i, k+\frac{1}{2}\right)=Q_{i} \psi_{e_{z \rho}}^{n}\left(i, k+\frac{1}{2}\right)+\frac{P_{i}}{\Delta \rho} \sum_{l} a(l) \\
& \cdot h_{\varphi}^{n+1 / 2}\left(i+l+\frac{1}{2}, k+\frac{1}{2}\right) \\
& h_{\rho}^{n+1 / 2}\left(i, k+\frac{1}{2}\right)=C P_{x}\left(i, k+\frac{1}{2}\right) h_{\rho}^{n}\left(i, k+\frac{1}{2}\right) \\
& +C Q_{x}\left(i, k+\frac{1}{2}\right)\left(\frac{\sum_{l} a(l) e_{\varphi}^{n+1 / 2}(i, k+l+1)}{\kappa_{k+1 / 2} \Delta z}\right. \\
& \left. \pm \frac{m}{i \Delta \rho} e_{z}^{n}\left(i, k+\frac{1}{2}\right)+\psi_{h_{\rho z}}^{n+1 / 2}\left(i, k+\frac{1}{2}\right)\right) \\
& \psi_{h_{\rho z}}^{n+1 / 2}\left(i, k+\frac{1}{2}\right)=Q_{k+1 / 2} \psi_{h_{\rho z}}^{n}\left(i, k+\frac{1}{2}\right)+\frac{P_{k+1 / 2}}{\Delta z} \\
& \cdot \sum_{l} a(l) e_{\varphi}^{n+1 / 2}(i, k+l+1)
\end{aligned}
$$

(13ai)

(13bii)

(13di)

$$
\begin{aligned}
& h_{\varphi}^{n+1 / 2}\left(i+\frac{1}{2}, k+\frac{1}{2}\right)=C P_{\varphi}\left(i+\frac{1}{2}, k+\frac{1}{2}\right) h_{\varphi}^{n}(i \\
& \left.+\frac{1}{2}, k+\frac{1}{2}\right)+C Q_{\varphi}\left(i+\frac{1}{2}, k+\frac{1}{2}\right) \\
& \cdot\left(\frac{\sum_{l} a(l) e_{z}^{n+1 / 2}(i+l+1, k+1 / 2)}{\kappa_{i+1 / 2} \Delta \rho}\right. \\
& -\frac{\sum_{l} a(l) e_{\rho}^{n}(i+1 / 2, k+l+1)}{\kappa_{i+1 / 2} \Delta z} \\
& \left.+\psi_{h_{\varphi \rho}}^{n+1 / 2}\left(i+\frac{1}{2}, k+\frac{1}{2}\right)-\psi_{h_{\varphi z}}^{n}\left(i+\frac{1}{2}, k+\frac{1}{2}\right)\right) \\
& \psi_{h_{\varphi z}}^{n+1 / 2}\left(i+\frac{1}{2}, k+\frac{1}{2}\right)=Q_{k+1 / 2} \psi_{h_{\varphi z}}^{n}\left(i+\frac{1}{2}, k+\frac{1}{2}\right) \\
& +\frac{P_{k+1 / 2}}{\Delta z} \sum_{l} a(l) e_{\rho}^{n}\left(i+\frac{1}{2}, k+l+1\right) \\
& \psi_{h_{\varphi \rho}}^{n+1 / 2}\left(i+\frac{1}{2}, k+\frac{1}{2}\right)=Q_{i+1 / 2} \psi_{h_{\varphi \rho}}^{n}\left(i+\frac{1}{2}, k+\frac{1}{2}\right) \\
& +\frac{P_{i+1 / 2}}{\Delta \rho} \sum_{l} a(l) e_{z}^{n+1 / 2}\left(i+l+1, k+\frac{1}{2}\right) \\
& h_{z}^{n+1 / 2}\left(i+\frac{1}{2}, k\right)=C P_{z}\left(i+\frac{1}{2}, k\right) h_{z}^{n}\left(i+\frac{1}{2}, k\right) \\
& +C Q_{z}\left(i+\frac{1}{2}, k\right)\left(\mp \frac{m e_{\rho}^{n+1 / 2}(i+1 / 2, k)}{(i+1 / 2) \Delta \rho}\right. \\
& -\frac{\sum_{l} a(l)(i+l+1) e_{\varphi}^{n}(i+l+1, k)}{\kappa_{i+1 / 2}(i+1 / 2) \Delta \rho} \\
& \left.-\psi_{h_{z \rho}}^{n}\left(i+\frac{1}{2}, k\right)\right) \\
& \psi_{h_{z \rho}}^{n+1 / 2}\left(i+\frac{1}{2}, k\right)=Q_{i+1 / 2} \psi_{h_{z \rho}}^{n}\left(i+\frac{1}{2}, k\right)+\frac{P_{i+1 / 2}}{\Delta \rho} \\
& \cdot \sum_{l} a(l) E_{y}^{n}(i+l+1, k) . \\
& e_{\rho}^{n+1}\left(i+\frac{1}{2}, k\right)=C A_{x}\left(i+\frac{1}{2}, k\right) e_{\rho}^{n+1 / 2}\left(i+\frac{1}{2}, k\right) \\
& +C B_{x}\left(i+\frac{1}{2}, k\right)\left( \pm \frac{m}{(i+1 / 2) \Delta \rho} h_{z}^{n+1 / 2}\left(i+\frac{1}{2}, k\right)\right. \\
& -\frac{1}{\kappa_{k} \Delta z} \sum_{l} a(l) h_{\varphi}^{n+1}\left(i+\frac{1}{2}, k+l+\frac{1}{2}\right) \\
& \left.-\psi_{e_{\rho z}}^{n+1}\left(i+\frac{1}{2}, k\right)\right) \\
& \psi_{e_{\rho z}}^{n+1}\left(i+\frac{1}{2}, k\right)=Q_{k} \psi_{e_{\rho z}}^{n+1 / 2}\left(i+\frac{1}{2}, k\right)+\frac{P_{k}}{\Delta z} \sum_{l} a(l) \\
& \cdot h_{\varphi}^{n+1}\left(i+\frac{1}{2}, k+l+\frac{1}{2}\right)
\end{aligned}
$$




$$
\begin{aligned}
& e_{\varphi}^{n+1}(i, k)=C A_{\varphi}(i, k) e_{\varphi}^{n+1 / 2}(i, k)+C B_{\varphi}(i, k) \\
& \cdot\left(\frac{1}{\kappa_{k} \Delta z} \sum_{l} a(l) h_{\rho}^{n+1 / 2}\left(i, k+l+\frac{1}{2}\right)\right. \\
& \quad-\frac{1}{\kappa_{i} \Delta \rho} \sum_{l} a(l) h_{z}^{n+1}\left(i+l+\frac{1}{2}, k\right)+\psi_{e_{\varphi z}^{n+1 / 2}}(i, k) \\
& \left.-\psi_{e_{\varphi \rho}^{n+1}}^{n}(i, k)\right)
\end{aligned}
$$$$
\psi_{e_{\varphi z}}^{n+1}(i, k)=Q_{k} \psi_{e_{\varphi z}^{n+1 / 2}}(i, k)+\frac{P_{k}}{\Delta z} \sum_{l} a(l) h_{\rho}^{n+1 / 2}(i, k
$$$$
\left.+l+\frac{1}{2}\right)
$$

(14bi)

$$
\begin{aligned}
& \psi_{e_{\varphi \rho}}^{n+1}(i, k)=Q_{i} \psi_{e_{\varphi \rho}}^{n+1 / 2}(i, k)+\frac{P_{i}}{\Delta \rho} \sum_{l} a(l) h_{z}^{n+1}(i+l \\
& \left.+\frac{1}{2}, k\right)
\end{aligned}
$$$$
e_{z}^{n+1}\left(i, k+\frac{1}{2}\right)=C A_{z}\left(i, k+\frac{1}{2}\right) e_{z}^{n+1 / 2}\left(i, k+\frac{1}{2}\right)
$$$$
+C B_{z}\left(i, k+\frac{1}{2}\right)
$$$$
\cdot\left(\frac{\sum_{l} a(l)(i+l+1 / 2) h_{\varphi}^{n+1 / 2}(i+l+1 / 2, k+1 / 2)}{i \kappa_{i} \Delta \rho}\right.
$$$$
\left.\mp \frac{m}{i \Delta \rho} h_{\rho}^{n+1}\left(i, k+\frac{1}{2}\right)+\psi_{e_{z \rho}}^{n+1 / 2}\left(i, k+\frac{1}{2}\right)\right)
$$$$
\psi_{e_{z \rho}}^{n+1}\left(i, k+\frac{1}{2}\right)=Q_{i} \psi_{e_{z \rho}}^{n+1 / 2}\left(i, k+\frac{1}{2}\right)+\frac{P_{i}}{\Delta \rho} \sum_{l} a(l)
$$$$
\cdot h_{\varphi}^{n+1 / 2}\left(i+l+\frac{1}{2}, k+\frac{1}{2}\right)
$$$$
h_{\rho}^{n+1}\left(i, k+\frac{1}{2}\right)=C P_{\rho}\left(i, k+\frac{1}{2}\right) h_{\rho}^{n+1 / 2}\left(i, k+\frac{1}{2}\right)
$$$$
+C Q_{\rho}\left(i, k+\frac{1}{2}\right)\left(\frac{\sum_{l} a(l) e_{\varphi}^{n+1 / 2}(i, k+l+1)}{\kappa_{k+1 / 2} \Delta z}\right.
$$$$
\left.\pm \frac{m}{i \Delta \rho} e_{z}^{n+1}\left(i, k+\frac{1}{2}\right)+\psi_{h_{\rho z}}^{n+1 / 2}\left(i, k+\frac{1}{2}\right)\right)
$$

$$
\begin{aligned}
& \psi_{h_{\rho z}}^{n+1}\left(i, k+\frac{1}{2}\right)=Q_{k+1 / 2} \psi_{h_{\rho z}}^{n+1 / 2}\left(i, k+\frac{1}{2}\right)+\frac{P_{k+1 / 2}}{\Delta z} \\
& \cdot \sum_{l} a(l) e_{\varphi}^{n+1 / 2}(i, k+l+1)
\end{aligned}
$$$$
h_{\varphi}^{n+1}\left(i+\frac{1}{2}, k+\frac{1}{2}\right)=C P_{\varphi}\left(i+\frac{1}{2}, k+\frac{1}{2}\right) h_{\varphi}^{n+1 / 2}(i
$$$$
\left.+\frac{1}{2}, k+\frac{1}{2}\right)+C Q_{\varphi}\left(i+\frac{1}{2}, k+\frac{1}{2}\right)
$$

$$
\begin{aligned}
& \cdot\left(\frac{\sum_{l} a(l) e_{z}^{n+1 / 2}(i+l+1, k+1 / 2)}{\Delta \rho}\right. \\
& -\frac{\sum_{l} a(l) e_{\rho}^{n+1}(i+1 / 2, k+l+1)}{\Delta z} \\
& \left.+\psi_{h_{\varphi \rho}}^{n+1 / 2}\left(i+\frac{1}{2}, k+\frac{1}{2}\right)-\psi_{h_{\varphi z}}^{n+1}\left(i+\frac{1}{2}, k+\frac{1}{2}\right)\right) \\
& \psi_{h_{\varphi z}}^{n+1}\left(i+\frac{1}{2}, k+\frac{1}{2}\right)=Q_{k+1 / 2} \psi_{h_{\varphi z}}^{n+1 / 2}\left(i+\frac{1}{2}, k+\frac{1}{2}\right) \\
& +\frac{P_{k+1 / 2}}{\Delta z} \sum_{l} a(l) e_{\rho}^{n+1}\left(i+\frac{1}{2}, k+l+1\right) \\
& \psi_{h_{\varphi \rho}}^{n+1}\left(i+\frac{1}{2}, k+\frac{1}{2}\right)=Q_{i+1 / 2} \psi_{h_{\varphi \rho}}^{n+1 / 2}\left(i+\frac{1}{2}, k+\frac{1}{2}\right) \\
& +\frac{P_{i+1 / 2}}{\Delta \rho} \sum_{l} a(l) e_{z}^{n+1 / 2}\left(i+l+1, k+\frac{1}{2}\right) \\
& h_{z}^{n+1}\left(i+\frac{1}{2}, k\right)=C P_{z}\left(i+\frac{1}{2}, k\right) h_{z}^{n+1 / 2}\left(i+\frac{1}{2}, k\right) \\
& +C Q_{z}\left(i+\frac{1}{2}, k\right)\left(\mp \frac{m e_{\rho}^{n+1 / 2}(i+1 / 2, k)}{(i+1 / 2) \Delta \rho}\right. \\
& -\frac{\sum_{l} a(l)(i+l+1) e_{\varphi}^{n+1}(i+l+1, k)}{\kappa_{i+1 / 2}(i+1 / 2) \Delta \rho} \\
& \left.-\psi_{h_{z \rho}}^{n+1}\left(i+\frac{1}{2}, k\right)\right) \\
& \psi_{h_{z \rho}}^{n+1}\left(i+\frac{1}{2}, k\right)=Q_{i+1 / 2} \psi_{h_{z \rho}}^{n+1 / 2}\left(i+\frac{1}{2}, k\right)+\frac{P_{i+1 / 2}}{\Delta \rho} \\
& \cdot \sum_{l} a(l) e_{\varphi}^{n+1}(i+l+1, k)
\end{aligned}
$$

where

$$
\begin{aligned}
& C A_{\rho}\left(i+\frac{1}{2}, k\right)=\frac{4 \varepsilon-\sigma \Delta t}{4 \varepsilon+\sigma \Delta t} \\
& C P_{\rho}\left(i, k+\frac{1}{2}\right)=\frac{4 \mu-\sigma_{m} \Delta t}{4 \mu+\sigma_{m} \Delta t} \\
& C B_{\rho}\left(i+\frac{1}{2}, k\right)=\frac{2 \Delta t}{4 \varepsilon+\sigma \Delta t} \\
& C Q_{\rho}\left(i, k+\frac{1}{2}\right)=\frac{2 \Delta t}{4 \mu+\sigma_{m} \Delta t} \\
& C A_{\varphi}(i, k)=\frac{4 \varepsilon-\sigma \Delta t}{4 \varepsilon+\sigma \Delta t} \\
& C P_{\varphi}\left(i+\frac{1}{2}, k+\frac{1}{2}\right)=\frac{4 \mu-\sigma_{m} \Delta t}{4 \mu+\sigma_{m} \Delta t} \\
& C B_{\varphi}(i, k)=\frac{2 \Delta t}{4 \varepsilon+\sigma \Delta t} \\
& C Q_{\varphi}\left(i+\frac{1}{2}, k+\frac{1}{2}\right)=\frac{2 \Delta t}{4 \mu+\sigma_{m} \Delta t}
\end{aligned}
$$




$$
\begin{aligned}
& C A_{z}\left(i, k+\frac{1}{2}\right)=\frac{4 \varepsilon-\sigma \Delta t}{4 \varepsilon+\sigma \Delta t} \\
& C P_{z}\left(i+\frac{1}{2}, k\right)=\frac{4 \mu-\sigma_{m} \Delta t}{4 \mu+\sigma_{m} \Delta t} \\
& C B_{z}\left(i, k+\frac{1}{2}\right)=\frac{2 \Delta t}{4 \varepsilon+\sigma \Delta t} \\
& C Q_{z}\left(i+\frac{1}{2}, k\right)=\frac{2 \Delta t}{4 \mu+\sigma_{m} \Delta t} \\
& P_{\xi}=\frac{\sigma_{\xi}}{\sigma_{\xi} \kappa_{\xi}+\kappa_{\xi}^{2} \alpha_{\xi}}\left(e^{-\left(\sigma_{\xi} / \kappa_{\xi}+\alpha_{\xi}\right)\left(\Delta t / 2 \varepsilon_{0}\right)}-1\right), \\
& Q_{\xi}=e^{-\left(\sigma_{\xi} / \kappa_{\xi}+\alpha_{\xi}\right)\left(\Delta t / 2 \varepsilon_{0}\right)},
\end{aligned}
$$

$$
\xi=i, k .
$$

Equations (13a)-(13fi) and (14a)-(14fi) can be also solved by the generalized Thomas method [5]. The value of $e_{z}$ is calculated the same as (11) and (12).

In the matched layer, the coefficients $\sigma_{i}$ and $\kappa_{i}$ are defined as follows $[12,13]$ :

$$
\begin{aligned}
& \sigma(\rho)=\sigma_{\max }\left(\frac{\rho}{d}\right)^{m} \\
& \kappa(\rho)=1+\left(\kappa_{\max }-1\right)\left(\frac{\rho}{d}\right)^{m},
\end{aligned}
$$

where $\rho$ is the distance from the spot in the matched layer to the interface between computational domain and matched layer, $d$ is the thickness of matched layer, and $m$ is a polynomial coefficient. $\sigma_{\max }$ is defined as follows:

$$
\begin{aligned}
\sigma_{\max } & =k \sigma_{\mathrm{opt}} \\
\sigma_{\mathrm{opt}} & =\frac{(m+1)}{150 \pi \sqrt{\varepsilon_{r}} \Delta},
\end{aligned}
$$

where $k=\sigma_{\max } / \sigma_{\mathrm{opt}}$ is positive and $\alpha$ is positive too.

\section{Numerical Results}

ADI-BOR-MRTD method has been tested by a metal ball and a metal cylinder with half-ball-hat. For comparison, they have been also calculated by FDTD and MRTD methods.

CPU is Intel(R) Core(TM) i3 $2.93 \mathrm{GHz}$; the memory bank is $1.93 \mathrm{~GB}$; the Mac OS is Microsoft Windows XP Professional; the operating system is Fortran 90 Complier.

5.1. The Ball. The radius of metal ball is 1 meter. The results are shown in Figure 2 and Table 2.

(1) FDTD: $\Delta x \times \Delta y \times \Delta z=2 \mathrm{~cm} \times 2 \mathrm{~cm} \times 2 \mathrm{~cm}, \Delta t=$ $3.33 \times 10^{-11} \mathrm{~s}$, and the cell lattice is $138 \times 138 \times 138$ with eight-cell-thick matched layer.

(2) MRTD: $\Delta x \times \Delta y \times \Delta z=10 \mathrm{~cm} \times 10 \mathrm{~cm} \times 10 \mathrm{~cm}, \Delta t=$ $11.11 \times 10^{-11} \mathrm{~s}$, and the cell lattice is $56 \times 56 \times 56$ with eight-cell-thick matched layer.

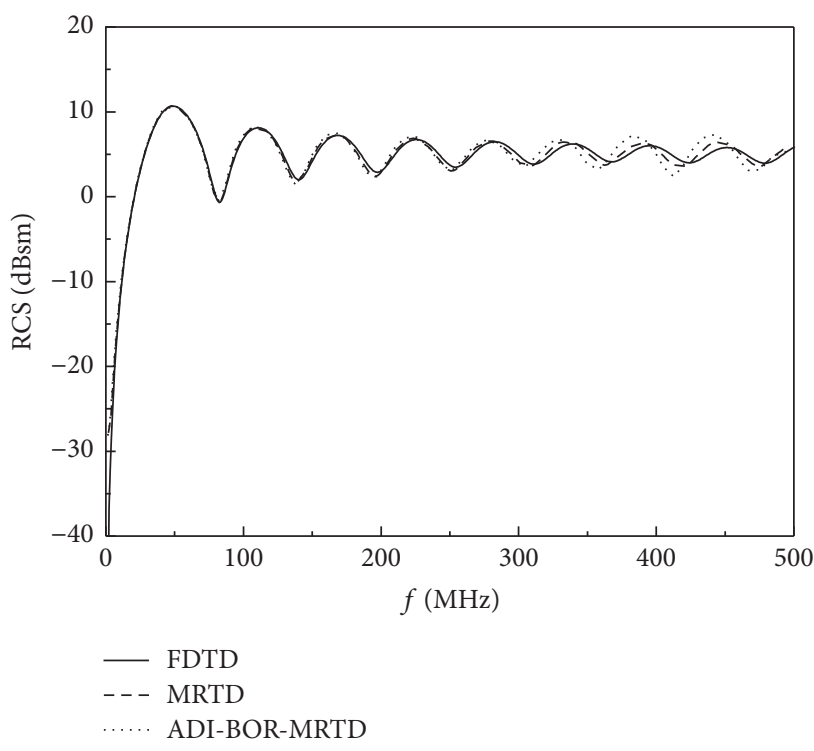

Figure 2: Single station RCS of metal ball.

TABLE 2: Comparison of the time and memory of CPU.

\begin{tabular}{lccc}
\hline & FDTD & MRTD & ADI-BOR-MRTD \\
\hline CPU time/s & 1652 & 5 & 0.4 \\
Memory/MB & 172.3 & 13.4 & 2.6
\end{tabular}

TABLE 3: Comparison of the time and memory of CPU.

\begin{tabular}{lccc}
\hline & FDTD & MRTD & ADI-BOR-MRTD \\
\hline CPU time/s & 1550 & 37 & 5 \\
Memory/MB & 161 & 13 & 0.23 \\
\hline
\end{tabular}

(3) ADI-BOR-MRTD: $\Delta \rho \times \Delta z=10 \mathrm{~cm} \times 10 \mathrm{~cm}, \Delta t=$ $22.22 \times 10^{-11} \mathrm{~s}$, the cell lattice is $28 \times 56$ with eight-cellthick matched layer, and the modulus range is $m=$ $0 \sim 16$.

Figure 2 shows that when the frequency is less than $500 \mathrm{MHz}$, the differences among three numerical results are less than $2 \mathrm{~dB}$, which validate the feasibility of the ADI-BORMRTD method. Moreover, Table 2 demonstrates that the ADI-BOR-MRTD method has taken less time and memory of CPU than the other two methods.

5.2. The Cylinder with Half-Ball-Hat. The metal cylinder with half-ball-hat is designed as Figure 3.

The results are shown as Figure 4 and Table 3.

(1) FDTD: $\Delta x \times \Delta y \times \Delta z=1 \mathrm{~cm} \times 1 \mathrm{~cm} \times 1 \mathrm{~cm}, \Delta t=$ $1.67 \times 10^{-11} \mathrm{~s}$, and the cell lattice is $96 \times 96 \times 266$ with eight-cell-thick matched layer.

(2) MRTD: $\Delta x \times \Delta y \times \Delta z=5 \mathrm{~cm} \times 5 \mathrm{~cm} \times 5 \mathrm{~cm}, \Delta t=$ $5.56 \times 10^{-11} \mathrm{~s}$, and the cell lattice is $48 \times 48 \times 82$ with eight-cell-thick matched layer. 


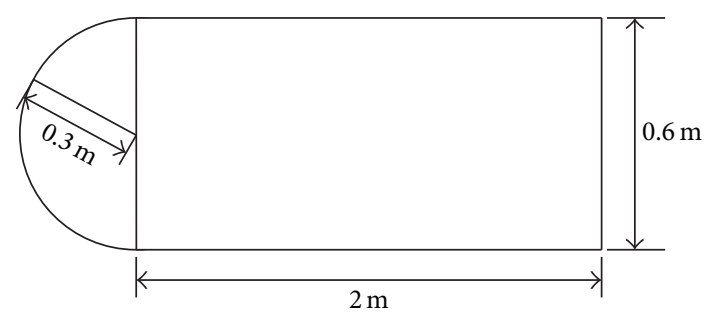

FIGURE 3: Structure of cylinder with half-ball-hat.

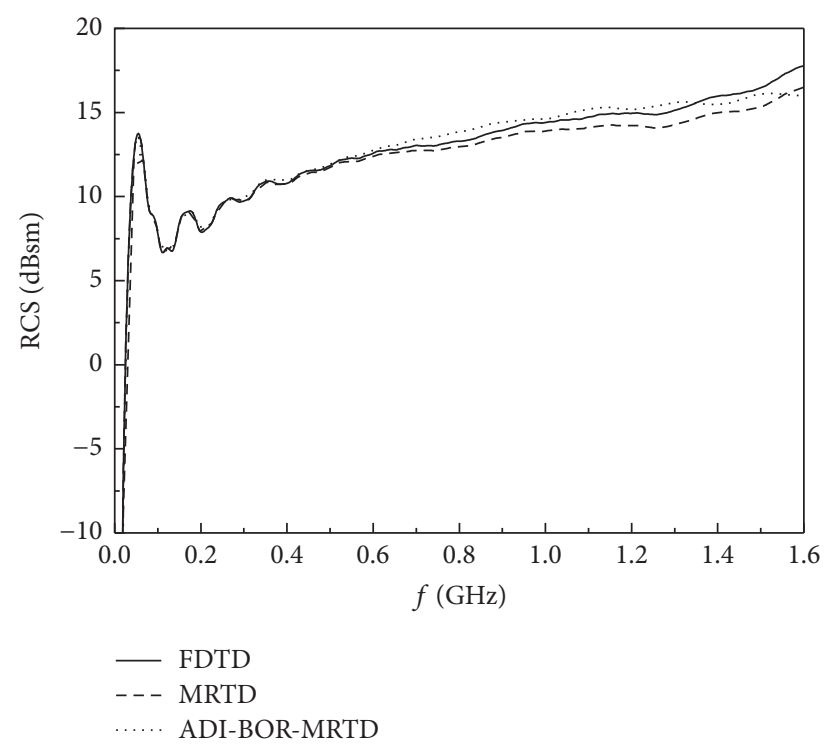

FIGURE 4: Single station RCS of metal cylinder with half-ball-hat.

(3) ADI-BOR-MRTD: $\Delta \rho \times \Delta z=5 \mathrm{~cm} \times 5 \mathrm{~cm}, \Delta t=$ $11.11 \times 10^{-11} \mathrm{~s}$, the cell lattice is $24 \times 82$ with eightcell-thick matched layer, and the modulus range is $m=0 \sim 16$.

From Figure 4 we can see that when the frequency is less than $1.5 \mathrm{GHz}$, the differences among three numerical results are less than $3 \mathrm{~dB}$ and the curves are similar. The results in Table 3 have also supported that ADI-BOR-MRTD method has taken less time and memory of CPU than the other two methods.

\section{Conclusion}

This paper has developed an ADI-BOR-MRTD algorithm. Furthermore, the CPML absorbing boundary condition is derived for ADI-BOR-MRTD algorithm. The simulated results suggest that the ADI-BOR-MRTD scheme can save more CPU time and memory than the FDTD and MRTD methods, which proves that the ADI-BOR-MRTD scheme is practicable, especially in the body of revolution case. In the next work, the method would be improved feasible for the frequency more than $500 \mathrm{MHz}$.

\section{Competing Interests}

The authors declare that they have no competing interests.

\section{References}

[1] M. Krumpholz and L. P. B. Katehi, "MRTD: new time-domain schemes based on multiresolution analysis," IEEE Transactions on Microwave Theory and Techniques, vol. 44, no. 4, pp. 555-571, 1996.

[2] S.-Y. Dai, Z.-S. Wu, and Y.-B. Xu, "Using the MRTD based on Daubechies scaling functions to solve the problem of electromagnetic scattering," Acta Physica Sinica, vol. 56, no. 2, pp. 786-790, 2007.

[3] D. W. Peaceman and J. Rachford, "The numerical solution of parabolic and elliptic differential equations," Journal of the Society for Industrial and Applied Mathematics, vol. 3, pp. 2841, 1955.

[4] Z. Chen and J. Zhang, "An unconditionally stable 3-D ADIMRTD method free of the CFL stability condition," IEEE Microwave and Wireless Components Letters, vol. 11, no. 8, pp. 349-351, 2001.

[5] L.-H. Wang and X.-L. Wu, "Analysis of the numerical dispersion of the ADI-MRTD," Modern Electronics Technique, vol. 11, no. 6, pp. 17-20, 2007.

[6] L. H. Wang, Application of MRTD and Its Improved Algorithms to Electromagnetic Scattering, Anhui University, 2007.

[7] W. Tang, Application of ADI-FDTD and Its Improved Algorithms to Electromagnetic Scattering, Xidian University, 2005.

[8] C. K. Chui, An Introduction to Wavelets, vol. 1 of Wavelet Analysis and Its Applications, Academic Press,, Boston, Mass, USA, 1992.

[9] I. Daubechies, Ten Lectures on Wavelets, vol. 61 of CBMS-NSF Regional Conference Series in Applied Mathematics, Society for Industrial and Applied Mathematics (SIAM), Philadelphia, Pa, USA, 1992.

[10] T. Namiki, "A New FDTD algorithm based on alternatingdirection implicit method," IEEE Transactions on Microwave Theory and Techniques, vol. 47, no. 10, pp. 2003-2007, 1999.

[11] Y. Liu, Y. Chen, P. Zhang, and X. Xu, "Implementation and application of a convolution PML using the MRTD algorithm," Journal of Electromagnetic Waves and Applications, vol. 28, no. 14, pp. 1736-1745, 2014.

[12] M. Kuzuoglu and R. Mittra, "Frequency dependence of the constitutive parameters of causal perfectly matched anisotropie absorbers," IEEE Microwave and Guided Wave Letters, vol. 6, no. 12, pp. 447-449, 1996.

[13] S. D. Gedney, G. Liu, J. A. Roden, and A. Zhu, "Perfectly matched layer media with CFS for an unconditionally stable ADI-FDTD method," IEEE Transactions on Antennas and Propagation, vol. 49, no. 11, pp. 1554-1559, 2001. 


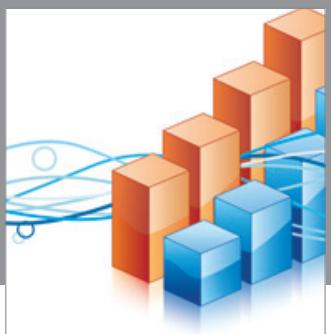

Advances in

Operations Research

vatem alat4

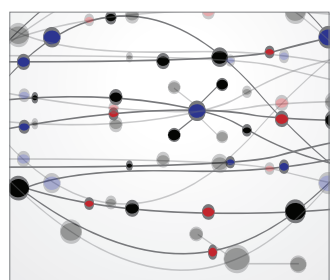

\section{The Scientific} World Journal
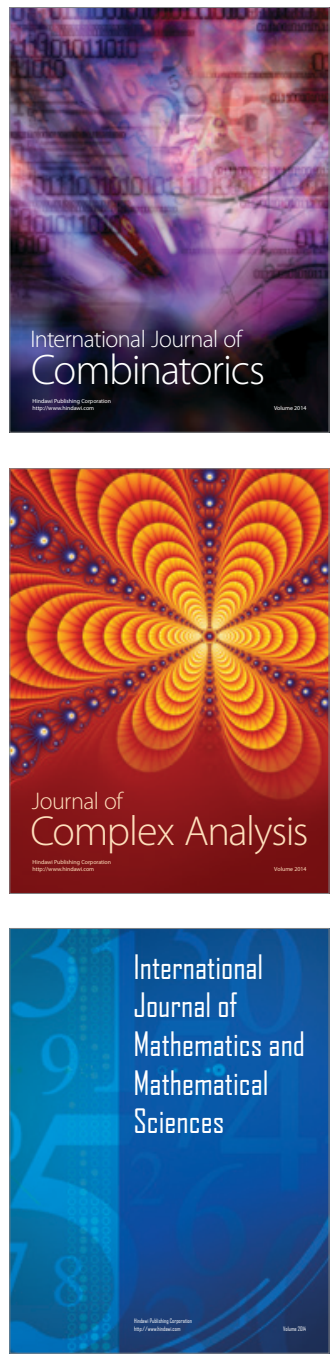
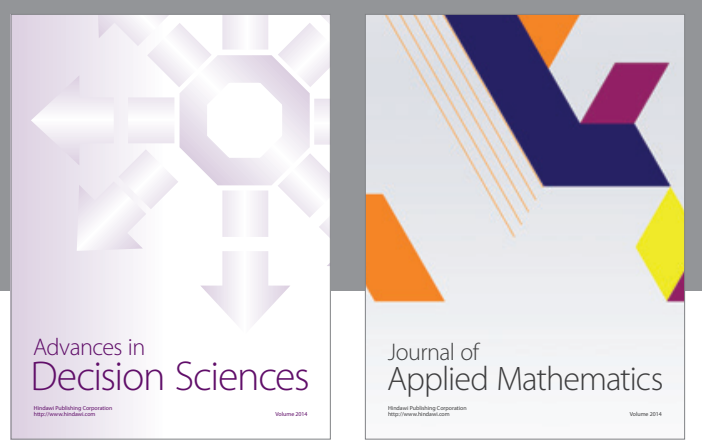

Algebra

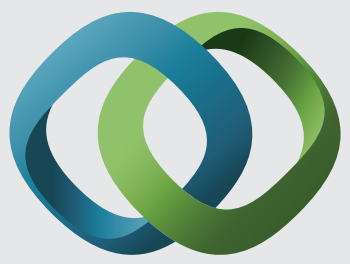

\section{Hindawi}

Submit your manuscripts at

https://www.hindawi.com
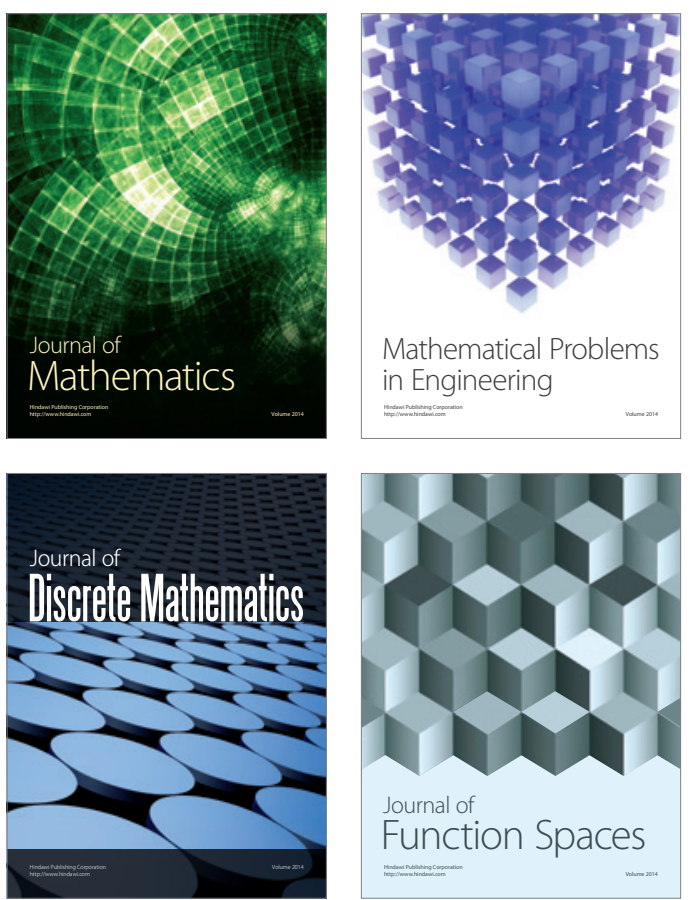

Mathematical Problems in Engineering
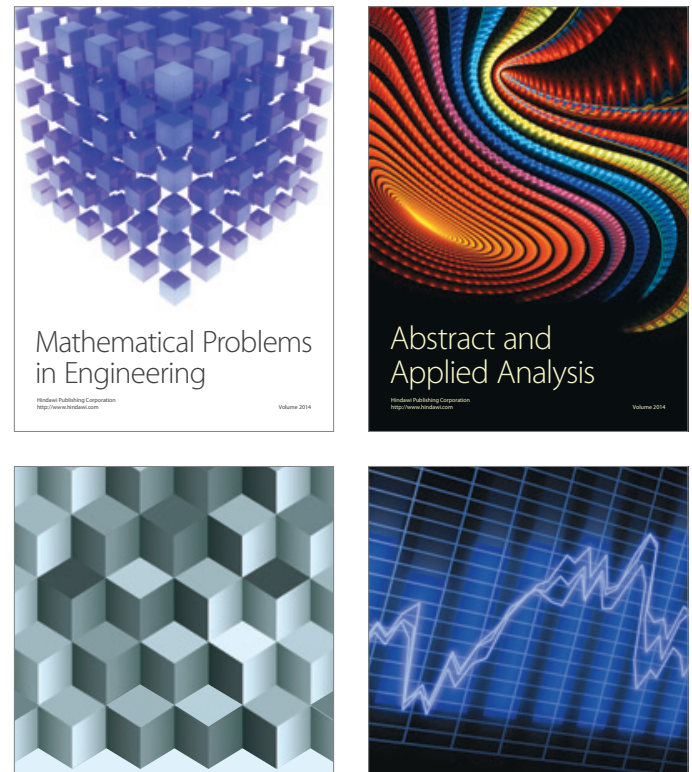

Journal of

Function Spaces

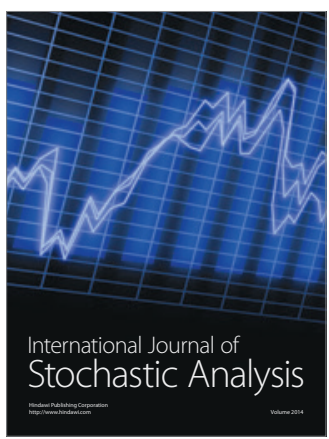

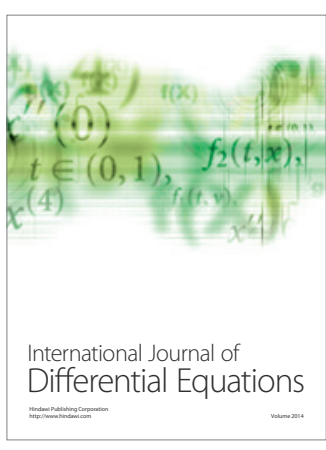
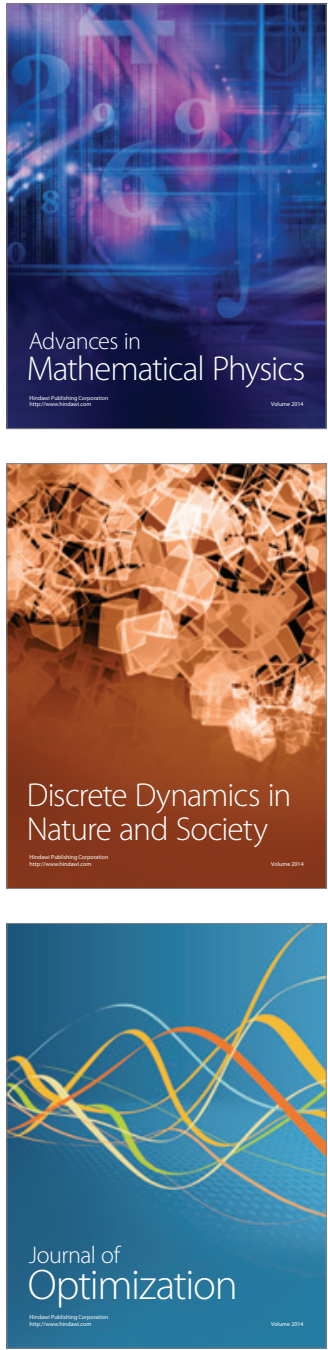